๑ Entomologica Fennica. 28 December 2001

\title{
Changes 1996-2000 in the list of Finnish insects
}

\author{
Hans Silfverberg
}

Silfverberg, H. 2001: Changes 1996-2000 in the list of Finnish insects. Entomol. Fennica 12: 217-243.

During the period 127 species have been deleted from the list of Finnish insects, and 555 species have been added to it. At the end of the year 2000, a total of 19977 insect species were recorded from Finland.

Hans Silfverberg, Zoological Museum, P.O. Box 17, FIN-00014 University of Helsinki,Finland; E-mail: hans.silfverberg@helsinki.fi.

Received 4 January 2001, accepted 10 September 2001

This paper is a sequel to a similar list published in 1996 (Silfverberg, Entomol. Fenn. 7: 39-49), which itself was a continuation of a series of lists published in 1921-1991 first by Hellén and then by Silfverberg. Insect species added to the fauna of Finland during the five-year period are listed, with references, as are those deleted from the fauna. A few previously unnoted changes have also been included.

For the new species the locality has generally been given, with its biogeographical province. If the species has been reported from many localities often only the provinces have been listed, or the abbreviation m.l. (= many localities) has been used. When Finland is mentioned as the locality, the original record did not give a more exact location.

Some other abbreviations or signs must also be explained. Thus f.d. $=$ false determination and sp.dist. $=$ distinct species (previously considered a variety, a synonym or such like); in literature references EF = Entomologica Fennica. A species enclosed in square brackets is considered accidentally imported, and has not been included in the species total. Again square brackets have been employed for the name used in the original record when different from the one used here. Other symbols are $*=$ new to science and $!=$ different from the check list mentioned for the order.

During the period covered by this list there have been 127 species deleted from the Finnish fauna, and 555 species added to it. At the end of year 2000, a total of 19977 insect species were known from Finland.

\section{Collembola}

\section{Deletions}

Ceratophysella armata (Nicol.) (f.d. = C. scotica (Carp. \& Ev.). Fjellberg 1998 Fauna Ent. Scand. 35: 40, 42

- longispina (Tullb.) (f.d.). ibid.: 41.

\section{Additions}

Hypogastrura neclecta (Börn.) (sp. dist.) Kb, Om, Ob. Fjellberg 1998: 32, 161.

Ceratophysella succinea Gisin Ab. ibid.: 45, 163. Anurida ellipsoides Stach Oa. ibid.: 84, 169.

Archaphorura serratotuberculata Stach Ab. ibid.: 101, 171.

Oligaphorura schoetti (Lie Pett.) Om. ibid.: 103, 171. Protaphorura subuliginata (Gisin) Ab, Ta, Tb. ibid.: 114, 173.

- pannonica (Hayb.) Ab. ibid.: 117, 173.

- fimata (Gisin) Ta. ibid.: 118, 173

Tullbergia arctica Wahlgr. Li. ibid.: 128, 175.

Paratullbergia callipygos (Börn.) Ab. ibid.: 131, 175. 
Mesaphorura critica Ellis Ab. ibid.: 141, 177.

- hylophila Rusek Ta. ibid.: 141, 177.

- tenuisensillata Rusek Ab,Ta,Li. ibid.: 141, 177.

*Folsomia palaearctica Pot. \& Bab. Li: Utsjoki Kevo ("S. Kopponen"). Potapov \& Babenko 2000 Eur. J. Ent. 97: 57.

Present number: $206-2+14=218$ spp.

\section{Ephemeroptera}

\section{Additions}

Potamanthus luteus (L.) Ka: Kotka, Ta: Kuusankoski. Vuori 1999 EF 10: 171.

Ephemera lineata Eat. Ta: Kuusankoski. Vuori \& Savolainen 1999 Sahlbergia 4: 53.

Heptageniaflava (Rost.) N: Vantaa. Hirvenoja 2000 Memoranda Soc. Fauna Flora Fenn. 76: 32.

Baetis tracheatus Keff. \& Mach. Lk: Kittilä. Savolainen \& Saura 1996 Sahlbergia 3: 28.

Present number: $51+4=55$ spp.

\section{Psocoptera}

\section{Additions}

Caecilius atricornis (McLachl.) Ab: Turku. Kanervo 1996 EF 7: 1.

Peripsocus subfasciatus (Ramb.) Ab: Turku. ibid.: 1. Amphigerontia intermedia (Tet.) Ab: Turku. Kanervo 1997 EF 8: 1.

Present number: $62+3=65$ spp.

\section{Hemiptera}

\section{Deletions}

Nysius groenlandicus (Zett.) (=N. ericae (Schill.), var.). Pericart 1998 Faune de France 84A: 217.

Aphalara pauli Login. (f.d. = A. ulicis Först.). Burckhardt \& Lawrence 1997 Ent. Scand. 28: 293.

Dysaphis aizenbergi (Shap.) (f.d. = Brachycaudus linariae Stroy.). Heikinheimo 1997 EF 7: 175.

\section{Additions}

Stenocranus major (Kirschb.) Ta: Jokioinen. Rinne \& Vasarainen 1998 EF 9: 1.

Zygina nigritarsis Rem. Ab: Korpo. Rinne 1996 EF 7: 1.

Aphalara ulicis Först. (pauli auct.). Finland. Burckhardt \& Lawrence 1997 Ent. Scand. 28: 295.

* freji Burckh. \& Lawr. (polygoni auct.). Finland. ibid.: 296.

Pemphigus spyrothecae Pass. N: Helsinki (Nuorteva). Heikinheimo 1997 EF 7: 162.

Callipterinella minutissima (Stroy.) N, Sa, Ob. ibid.: 162.

Tuberculatus querceus (Kalt.) N: Vantaa. ibid.: 164.

Thripsaphis caespitosae Oss. Lk: Sodankylä. ibid.: 165.

- brevicornis Oss. Ta: Janakkala (V.Vikberg). ibid.: 165.

Iziphya bufo (Walk.) N: Tvärminne (A.Albrecht). ibid.: 165.

Chaitophorus ramicola (Börn.) Ab, N,Ta.ibid.: 167.

[Rhopalosiphum rufiabdominale (Sas.) St: Noormarkku. ibid.: 168]

Melanaphis elisabethae (Oss.) $N$ : Vantaa, Sa: Mikkeli. ibid.: 168.

Schizaphis agrostis HRL Sa, Oa, Om, Ok, Ob. ibid.: 168.

- dubia Hucul. N: Vantaa. ibid.: 169.

- jaroslavi (Mordv.) N, Kb, Ks. ibid.: 170

- caricis (Schout.) Ab, N, St, Ta, Om, Ks. ibid.: 172.

Aphis avicularis (L.) N: Vantaa. ibid.: 173.

- longirostrata HRL N, St. ibid.: 173.

Brachycaudus linariae Str. N: Vantaa. ibid.: 175.

Myzus certus (Walk.) Al: Jomala, $N$ : Vantaa. ibid.: 177.

Present number: $1359-3+20=1376$ spp.

\section{Coleoptera}

\section{Deletions}

Ochthebius rugulosus Woll. (f.d. = O. alpinus (Ien.) \& O. hungaricus Endr.-Y.) Jäch 1998 Koleopterol. Rundschau 68: 179-180. 
Ptilium affine Er. (f.d. = P. exaratum (Allib.)) Rutanen 1996 EF 7: 3.

Bledius bosnicus Bernh. (f.d. = B. erraticus Er.) Clayhills 1996 EF 7: 68.

Globicornis corticalis (f.d. = G. emarginata (Gyll.)) Mannerkoski 1996 EF 7: 3.

Dasytes nigrocyaneus Muls. \& Rey (f.d. $=D$. obscurus Gyll.) Mannerkoski 1996: 3.

Epuraea melanocephala $($ Marsh.) (f.d. = Nitidula rufipes (L.)) Mannerkoski 1996: 3.

- concurrens Sjöb. (= E. hilleri Rtt. (fageticola Aud.)). Kirejtshuk 1997 Zoosyst. Ross. 6: 259.

Hyperaspis reppensis (Hbst.) (f.d. = H. pseudopustulata Muls. Clayhills 2000 EF 11: 111.

Cis castaneus Mell. (f.d. = Ennearthron cornutum (Gyll.)) Mannerkoski 1996: 3.

Anthicus axillaris Schm. (f.d. = A. flavipes (Panz.)) Mannerkoski 1996: 2.

Liophloeus lentus Germ. (f.d. = L. tessulatus (Müll.)). Mannerkoski 1999 EF 10: 5.

Hypera venusta (F.) (f.d. =H. meles (F.)) Mannerkoski 1996: 3.

Anthonomus pomorum (L.) (f.d.) Mannerkoski 1996: 2.

- ulmi (DeG.) (f.d. = A. undulatus (Gyll.)) Mannerkoski 1996: 3.

- rufus (Gyll.) (f.d. = A. conspersus Desbr.) Mannerkoski 1996: 3.

Ceutorhynchus pyrrhorhynchus (Marsh.) (f.d. = C. floralis (Payk.)) Mannerkoski 1996: 3.

- trimaculatus (F.) (f.d. = C. litura (F.)) Mannerkoski 1996: 3.

- melanostictus (Marsh.) (f.d. = C. arquatus (Hbst.)). Clayhills 1999 EF 10: 5.

Phloeotribus rhododactylus (Marsh.) (f.d.) Heliövaara et al. 1998 Univ. Helsinki Dept. Appl. Zool. Rep. 25: 86.

\section{Additions}

Bembidion azurescens (D.T.) N: Helsinki. Westman et al. 2000 Sahlbergia 5: 39.

Ochthebius alpinus (Ieniştea) (rugulosus auct. nec Woll.) N, Ta, Sb, Kb, Om, Ob, Lk. Jäch 1998 Koleopterol. Rundschau 68: 179

- hungaricus Endr.-Y. (rugulosus auct. nec Woll.) $A b, N$. ibid.: 180.

*Baranowskiella ehnstromi Sörens. Ab: Pargas (T.
Clayhills), Ekenäs Bromarv (P. Rassi \& I. Rutanen). Clayhills 1993 EF 4: 17 [“Nanosella sp.']; Sörensson 1997 System. Entomol. 22: 271.

Philonthus micantoides Ben. \& Lohse Ab: Korpo Jurmo. Clayhills et al. 2000 Metsähall. Luonnonsuoj.julk. A 113: 77.

Velleius dilatatus (F.) Ab: Dragsfjärd Örö (J. Kullberg). Forstén et al. 2000 Memoranda Soc. Fauna Flora Fenn. 76: 62.

Lesteva punctata Er. Ta: Somero (E. Helve \& R. Iivarinen). Forstén et al. 1998 Memoranda Soc. Fauna Flora Fenn. 74: 90. Iivarinen 1999 EF 10: 2.

Bledius erraticus Er. (bosnicus auct.) $\mathrm{Ks}, \mathrm{Li}$. Clayhills 1996 EF 7: 68.

Mycetoporus glaber (Sperk) Ab: Pargas (T. Clayhills). Schülke \& Kocian 2000 Entomol. B1. 96: 94.

Atheta (Alaobia) pandionis Scheerp. Ok: Puolanka \& Suomussalmi (R. Leinonen). Rutanen \& Kashevarov 1997 Finn. Environm. 124: 288. Rutanen \& Leinonen 1999 Sahlbergia 4: 56.

Oligota inexspectata Will. Ab: Dragsfjärd Örö. Clayhills et al. 2000: 52.

Platysoma compressum (Hbst.) Ka: Virolahti (J. Muona). Mannerkoski 1999 EF 10: 2.

Oxythyrea funesta (Poda) Sa: Imatra (E. Helve). Forstén et al. 1997 Memoranda Soc. Fauna Flora Fenn. 73: 66.

Limnichus sericeus (Duft.) N: Siuntio, Kb: Polvijärvi (M. Pohjola). Mannerkoski 1996 EF 7: 1.

[Lyctus africanus Lesne] Southern Finland. Muona 2000 EF 11: 2.

Anthocomus rufus (Hbst.) Ab: Korpo Jurmo. Clayhills et al. 2000: 51.

Atomaria lapponica Johns. Ab: Sammatti (J. Sahlberg), Ta: Korpilahti (J. Sahlberg). Clayhills 1999 EF 10: 2.

— strandi Johns. Ab: Karislojo (J. Sahlberg), $N$ : Helsinki (J. Sahlberg). ibid.: 2.

Hyperaspis pseudopustulata Muls. (reppensis auct.) $\mathrm{Ab}$ - Om (not as in map). Clayhills 2000 EF 11: 111.

Corticaria pineti Lohse Finland. Rassi 1993 WWF Suom. Rah. Rap. 6: 99.

[Stromatium fulvum (Vill.)] Finland. Muona 2000 EF 11: 2 .

Leptinotarsa decemlineata (Say) N, Ka, Ta, Sa, $K l, K b$. Tomminen 1999 Kasvinsuojelulehti 
32: 4. Mikkola 1999 Baptria 24: 101.

[Otiorhynchus corruptor (Hast)] N: Hangö. Silfverberg 2000 EF 11: 2.

Gnathotrichus materiarius (Fitch) $N$ : Vantaa. Valkama et al. 1997 EF 8: 155; Valkama et al. 1998 EF 8: 193.

Pityophthorus morosovi Spess. Sa: Sulkava. Heliövaara et al. 1998 Univ. Helsinki Dept. Appl. Zool. Rep. 25: 85.

Present number: $3629-19+22=3632$ spp.

\section{Trichoptera}

\section{Additions}

Allotrichia pallicornis (Eat.) Ka: Kotka (P. Sundell \& J. Kullberg). Salmela 2000 Diamina 9: 5.

Hydroptila vectis Curt. Ob: Simo, Li: Inari. Mattila \& Salmela 1999 Diamina 8: 1.

— dampfi Ulm. Ta: Toijala \& Lempäälä. Turunen 1999 EF 10: 2.

* Oxyethira tamperensis Mal. Ta: Tampere (H. Turunen \& E. Saarela). Malicky 1999 Braueria 26: 44; Turunen 2000 Diamina 9: 3.

- boreella Sv. \& Tj. Li: Inari. Laasonen 2000 EF 11: 2.

Crunoecia irrorata (Curt.) Ab, St, Ta, Oa. Saarela 1997 Diamina 6: 13. Laasonen et al. $1998 \mathrm{EF}$ 9: 135. Järventausta 1999 Diamina 8: 15. Saarela 1999 EF 10: 2.

Apatania stylata Navás Ta: Valkeakoski (K. Järventausta). Laasonen et al. 1998: 134.

Limnephilus hirsutus (Pict.) Ab: Korpo Elvsö. Leivo 1996 Diamina 5: 28. Laasonen et al. 1998: 127. Salokannel 1999 EF 10: 2.

Present number: $205+8=213$ spp.

\section{Lepidoptera}

Huldén, L. (ed.) 2000. Suomen suurperhosatlas. Finlands storfjärilsatlas. Atlas of Finnish Macrolepidoptera. Helsinki, 328 pp.

\section{Deletions}

Synanthedon herzi (Spat. \& Gorb.) (f.d.). Kerppola
1997 Baptria 22: 61

Hadena bicruris (Hufn.) (f.d. = capsincola $($ D. \& Sch.)). Kullberg 1999 Baptria 24: 118.

\section{Species reported but later considered incorrect}

Euxoa montivaga Fib. Ab, N, Ka. Pöyry 2000 Baptria 25: 73. Huldén 2000: 284.

\section{Additions}

Ectoedemia longicaudella Klim. Finland. Vuorinen 1998 Baptria 23: 112.

- turbidella (Zell.) N: Espoo (M. Tyni) Kaila 1998 Baptria 23: 113.

Phyllonorycter pyrifoliellus (Geras.) Sa: Lappeenranta. Saarela 1996 Diamina 5: 12. Kerppola 1997 Baptria 22: 51.

Zelleria hepariella Staint. Al (K. Saloranta), Ab: Dragsfjärd Örö (A. \& J. Kullberg). Vaalamo 1998 Baptria 23: 113. Forstén et al. 2000 Memoranda Soc. Fauna Flora Fenn. 76: 62.

Ypsolopha chazariella (Mann) N: Helsinki. Nupponen \& Nupponen 1996 Baptria 21: 33.

- sarmaticella (Rebel) Sa: Joutseno. Huusko \& Malinen 1996 Diamina 5: 22. Kerppola 1997: 52.

Acrolepia autumnitella Curt. Ab: Korpo Jurmo. Kaitila 1998 Baptria 23: 113.

Agonopterix subpropinquella Ka: Anjalankoski (H. Luoma). Kaitila 2000 Baptria 25: 74.

*Elachista ripula Kaila Li: Utsjoki (E. Linnaluoto). Kaila 1998 EF 8: 221.

Coleophora limosipennella (Dup.) Al: Finström (E. Laasonen). Kerppola 1997: 54.

— gallipennella (Hübn.) Ab: Dragsfjärd Örö (J. Kullberg), $N$ : Ekenäs Hästö (J. Itämies). Kullberg 1998 Baptria 23: 113.

- nutantella Mühl. \& Fr. N: Kirkkonummi. Junnilainen 1998 Baptria 23: 113.

Mompha langiella (Hübn.) Al: Eckerö. Nupponen 1997 Baptria 22: 1.

Monochroa niphognatha Ab: Turku. Winqvist 2000 Baptria 25: 74.

Athrips amoenella (Frey) Ta: Heinola, Lammi, Hämeenlinna. Kaila \& Kerppola 1995 Baptria 20: 98 [Athrips sp.]. Svensson 1996 Entomol. Tidskr. 117: 53. Kerppola 1997: 56. Mutanen 
et al. 1998: 56.

Scrobipalpula diffluella (Frey) Ks: Kuusamo Liikasenvaara (J. Kyrki). Huener \& Karsholt 1998 Nota Lepidopterol. 21: 55.

Helcystogramma lutatellum (H.-S.) Ab: Dragsfjärd Örö, $N$ : Inkoo. Kullberg 2000 Baptria 25: 133. Forstén et al. 2000 Memoranda Soc. Fauna Flora Fenn. 76: 62.

Neosphaleroptera nubilana (Hübn.) Al: Maarianhamina. Nupponen 1997: 1.

*Exapate bicuspidella Bruun \& Krog. Ks: Kuusamo. Bruun \& Krogerus 1996 EF 7: 77.

Lobesia virulenta Bae \& Komai Al-Ob. Kaitila 2000 Baptria 25: 74.

Eucosma jaceana (H.-S.) Ka: Koivuluoto (J. Kullberg \& P. Sundell). Pöyry 2000: 75.

Ancylis achatana (D. \& Sch.) Ab: Rymättylä (Vuorinen). Kerppola 1997: 58.

Cydia fagiglandana (Zell.) Ab: Dragsfjärd Örö. Kullberg 1998 Baptria 23: 113. Kullberg 1999 Baptria 24: 152.

Epermenia falciformis (Haw.) $\mathrm{Al}, \mathrm{N}, \mathrm{Ka}, \mathrm{Ta}, \mathrm{Ok}$, Ob. Laasonen \& Laasonen 1998 Baptria 23: 120.

Psorosa nucleolella (Möschl.) N: Borgå Munkby. Landtman 1999 Baptria 24: 114.

Phycitodes lacteella (Rothsch.) N: Kirkkonummi. Junnilainen 2000 Baptria 25: 75.

Vitula edmandsii (Pack.) N: Tammisaari (K. Mikkola). Kullberg 1999 Baptria 24: 114.

Loxostege turbidalis (Tr.) Ka: Virolahti (J. Kullberg \& M. Kuisma). Kullberg 2000 Baptria 25: 136 .

Palpita vitrealis (Rossi) (unionalis (Hübn.)) Ka: Virolahti. Savela 1999 Baptria 24: 116. Kaitila 2000 Baptria 25: 74.

Dolicharthria punctalis (D. \& Sch.) Ab: Dragsfjärd Örö. Kullberg 1997 Baptria 22: 135. Forstén et al. 2000: 62.

Cupido (Everes) alcetas (Hoffmgg.) Sa: Imatra (H. Nurmi). Kullberg 1996 Eläinmaailma 18(4):58. Huldén 2000: 56. Kolev \& Kullberg 2000 Baptria 25: 157.

[Morpho peleides Koll. N: Helsinki (M. Honkanen). Holmberg 1999 Baptria 24: 177.]

Cyclophora linearia (Hübn.) Ab: Dragsfjärd Örö (J. Kullberg), N: Vantaa (T. Salin). Mikkola 1999 Baptria 24: 101; Kullberg 1999 Baptria 24: 149. Kaitila et al. 1999 Baptria 24: 185. Huldén 2000: 83.

Eupithecia distinctaria H.-S. Ab: Dragsfjärd Örö.
Kullberg 2000 Baptria 25: 1. Huldén 2000: 125. (Chloroclystis v-ata) * relicta Krog. Ab: Karislojo. Krogerus 1996 EF 7: 63. Huldén 2000: 292.

Odonestis pruni (L.) Ka: Virolahti. Kullberg 1999 Eläinmaailma 21(10):9. Huldén 2000: 159.

Cerura erminea (Esp.) Finland. Mikkola 2000 Suomen Luonto 59(9):42.

Cucullia fraterna Butl. $N$ : Elimäki. Baptria 22: 101. Huldén 2000: 209.

Hoplodrina ambigua (D. \& Sch.) N: Hangö. Baptria 22: 101. Huldén 2000: 217.

Mormo maura (L.) Ka: Kotka (T. Nikki). Mikkola 1997 EF 8: 132. Repo \& Kullberg 1998 Baptria 23: 135. Huldén 2000: 296.

Dicycla oo (L.) N: Helsinki (L. Öberg) \& Sipoo (M. Varesvuo). Kaila 1998 Baptria 23: 113. Repo \& Kullberg 1998: 136. Kaitila et al. 1999: 197. Huldén 2000: 222.

Agrochola lychnidis (D. \& Sch.) Al: Kökar, $N$ : Esbo. Grotenfelt 1999 Baptria 24: 114. Huldén 2000: 226.

* Discestra [Hadula] farnhami palaearctica Hacker. Ob: Rovaniemi. Hacker 1998 Esperiana 6: 689. Kaila \& Kullberg 2000 Baptria 25: 74. Huldén 2000: 297.

Lacanobia splendens (Hübn.) N: Kirkkonummi (Sundell \& Elo). Repo \& Kullberg 1997 Baptria 22: 43. Sundell 1997 Baptria 22: 46. Huldén 2000: 253.

Hadena luteago (D. \& Sch.) N: Pernå (B. G. Kumlander), Ka: Kotka, Hamina \& Virolahti. Repo \& Kullberg 1997: 43. Silvonen 1998 Baptria 23: 22. Repo \& Kullberg 1998: 138. Huldén 2000: 250.

- capsincola (D. \& Sch.) (bicruris auct.) Al-Om. Kullberg 1999 Baptria 24: 174. Huldén 2000: 253.

Mythimna l-album (L.) Ab: Kustavi (P. Grotenfelt); $N$ : Hanko (J. \& A. Kullberg) \& Helsinki (H. Koski). Kaitila et al. 1999: 185. Huldén 2000: 261.

Noctua interjecta Hübn. N: Hanko (P. Koskinen); Ka: Kotka (T. Nikki). Mikkola 1997 EF 8: 132. Repo \& Kullberg 1998: 138. Huldén 2000: 270.

Xestia atrata (Morr.) Ks: Kuusamo (P. Sundell). Sundell 2000 Baptria 25: 77. Huldén 2000: 276. Sundell \& Lundsten 2000 Baptria 25: 92. Forstén et al. 2000: 62.

- agathina (Dup.) Ab: Kustavi (P. Grotenfelt). Kaitila et al. 1999: 185. Huldén 2000: 279. 
Euxoa eruta (Hübn.) Finland. Kullberg 1999 Baptria 24: 118. Kullberg 2000 Baptria 25: 73. Huldén 2000: 284.

Euplagia quadripunctaria (Poda) Ka: Karhula (L. Solanterä). Luukkonen 2000 Baptria 25: 75. Huldén 2000: 181.

Present number: $2378-2+50=2426$ spp.

\section{Diptera}

\section{Deletions}

Bezzia xanthocephala Goetgh. (= B. leucogaster (Zett.). Szadziewski 1986 Polskie Pismo Entomol. 56: 91.

Cheilosia honesta Rond. (= C. barbata Loew). Claussen \& Thompson 1996 Stud. dipterol. 3: 278.

- nasutula Beck. (= C. vicina (Zett.)). Lucas, Nielsen \& Ståhls 1995 Internat. Journ. Dipterol. Res. 6: 3.

Eristalis vallei (Kan.) (= E.fratercula (Zett.)). Nielsen 1995 Intern. J. Dipterol. Res. 6: 130.

Hydrellia chrysostoma (Meig.) (= H. griseola (Fall.)). Zatwarnicki 1988 Polskie Pismo Entomol. 58: 598.

Chamaepsila nigromaculata $(\mathrm{Strobl})(=$ C. bicolor (Meig.)). Carles-Tolrá 1995 Graellsia 49: 93.

Phytobia betulae (Kangas) (= P. cambii (Hend.). Tschirnhaus 1992 Decheniana Beih. 31: 480.

Liriomyza pedestris Hend. (= L. richteri Her.). Tschirnhaus 1981 Spixiana Suppl. 6: 32.

Stiphrosoma [Anthomyza] cingulatum (Halid.) (f.d. = S. laetum (Meig.)) Roháček 1996 Europ. J. Entomol. 93: 119.

Chlorops figuratus Zett. (f.d. = C. zonulatus Wahlgr.). Nartshuk 1998 EF 9: 154.

— geminatus Meig. (f.d. =C. zonulatus Wahlgr.). ibid.: 154.

- triangularis Beck. (= C. planifrons Loew, var.). ibid.: 154.

Peribaea setinervis (Thoms.) (fissicornis (Strobl)) (f.d. = P. hertingi And.). Andersen 1996 Fauna Ent. Scand. 33: 72.

Entomophaga [Ceromya] nigrohalterata Vill. (doubtful record), ibid.: 53.

Entomophaga exoleta (Meig.) (f.d.). ibid.: 55. Aphantorhaphopsis [Ceranthia] selecta (Pand.) (f.d.). ibid.: 111.

Siphona cristata $($ F.) = geniculata (DeG.). ibid.: 94 .

\section{Additions}

Psectrocladius barbatipes Kieff. Lk: Sodankylä Lokka. Hirvenoja 1998 Oulanka rep. 18: 38.

Chironomus borokensis Kerk. et al. Tb: Jyväskylä (L. Paasivirta). Wülker 1999 Stud. Dipterol. 6: 426.

- muratensis Ryser et al. Ta: Vanajavesi, Tb: Jyväskylä. ibid.: 426.

- sororius Wülk. Oa: Nurmo/ Seinäjoki (E. Koskenniemi). ibid.: 426.

- acutiventris Wülk. et al. Oa: Seinäjoki (E. Koskenniemi). ibid.: 428.

— melanotus Keyl Lk: Sodankylä. ibid.: 429.

- heteropilicornis Wülk. Kyrkösjärvi, Liikapuro, Vatianjärvi, Venetjärvi. ibid.: 429.

*_esai Wülk. Oa: Nurmo \& Jalasjärvi (E. Koskenniemi), Tb: Laukaa (L. Paasivirta). Wülker 1997 EF 8: 172. Wülker 1999: 429.

- melanescens Keyl Oa: Nurmo (E. Koskenniemi). Wülker 1999: 432.

- macani Freem. Venetjärvi. ibid.: 433.

*_ brevidentatus Hirv. \& Mich. Lk: Sodankylä Lokka. Hirvenoja \& Michailova 1998 EF 9: 226.

*_ improvidus Hirv. Lk: Sodankylä Lokka. Hirvenoja 1998: 45.

*__coaetaneus Hirv. Lk: Sodankylä Lokka. ibid.: 53.

Bolitophila austriaca Meyer Kb: Ilomantsi. Polevoi 1995 Karel. Biosph. Res. Stud., p.162.

*_limitis Polev. Kb: Ilomantsi. Polevoi 1996 Zoosyst. Ross. 4: 177.

- nigrolineata Landr. Kb: Ilomantsi. Polevoi 1995: 162. Polevoi 1996: 179.

- obscurior Stack. Kb: Ilomantsi. Polevoi 1995: 162. Polevoi 1996: 179.

Neoplatyura flava (Macq.) Kb: Ilomantsi. Polevoi 1995: 163. Polevoi 1996: 181.

Orfelia falcata Zaitz. Kb: Ilomantsi. Polevoi 1996: 181.

*Diadocidia trispinosa Polev. Kb: Ilomantsi. ibid.: 180.

Leptomorphus quadrimaculatus Mats Polevoi 1995: 163.

Paratinia sciarina Mik Kb: Ilomantsi. ibid.: 163. Sciophila buxtoni Freem. Kb: Ilomantsi. ibid.: 163. 
- quadriterga Huts. Kb: Ilomantsi. ibid.: 163.

Boletina dissipata Plass. Al: Lumparland. Hedmark 1998 Entomol. Tidskr. 119: 6.

Coelophthinia thoracica (Winn.) Al: Lumparland, Kb: Ilomantsi. Hedmark 1998: 7. Polevoi 1995: 164.

Coelosia bicornis Stack. Ob: Rovaniemi Pisavaara (R. Tuomikoski). Söli 1997 Entomol. Scand., Suppl. 50: 134.

Palaeodocosia janickii (Dziedz.) Al: Lumparland. Hedmark 1998: 8.

Ectrepesthoneura referta Plassm. Kb: Ilomantsi. Polevoi 1995: 164.

Leia crucigera Zett. Al: Lumparland. Hedmark 1998: 8

Mycetophila assimilis Mat. Kb: Ilomantsi. Polevoi 1995: 164.

- brevitarsata (Lašt.) $N$ : Esbo, $K b$ : Ilomantsi. Laštovka 1963 Čas. českols. Spol. Entomol. 60: 320. Polevoi 1995: 164.

— estonica Kur. Kb: Ilomantsi. Polevoi 1995: 164.

- lunata Meig. Kb: Ilomantsi. ibid.: 164 (previously included with doubt).

- stricklandi Laff. Kb: Ilomantsi. ibid.: 164.

Phronia gagnei Chandl. Kb: Ilomantsi. ibid.: 165.

Zygomyia pseudohumeralis Casp. Kb: Ilomantsi. ibid.: 165.

Allodia adunca Zaitz. Kb: Ilomantsi. ibid.: 165.

Anatella aquila Zaitz. Kb: Ilomantsi. ibid.: 165.

— ciliata Winn. Kb: Ilomantsi. ibid.: 165.

- flavomaculata Edw. Kb: Ilomantsi. ibid.: 165.

- lenis Dziedz. Kb: Ilomantsi. ibid.: 165.

- maritima Ostrov. Kb: Ilomantsi. ibid.: 165.

- setigera Edw. Kb: Ilomantsi. ibid.: 165.

— turi Dziedz. Kb: Ilomantsi. ibid.: 165.

- novata Dziedz. Al: Lumparland. Hedmark 2000 Entomol. Tidskr. 121: 80.

Brevicornu bellum (Joh.) Kb: Ilomantsi. Polevoi 1995: 165.

— foliatum Edw. Kb: Ilomantsi. ibid.: 166.

- setulosum Zaitz. Kb: Ilomantsi. ibid.: 166.

Cordyla parvipalpis Edw. Al: Lumparland, $K b$ : Ilomantsi. Hedmark 1998: 8. Polevoi 1995: 166.

— sixi (Barendr.) Kb: Ilomantsi. ibid.: 166.

Exechiopsis sagittata Lašt. \& Mat. Kb: Ilomantsi. ibid.: 166.

Pseudexechia aurivernica Chandl. Kb: Ilomantsi. ibid.: 166.

Scaptosciara curviforceps (Buk. \& Leng.) (myrmecophila Frey) Ab: Vichtis, $N$ : Helsingfors.
Frey 1948 Notulae Entomol. 27: 87; Menzel, Mohrig \& Báez 1996 Vieraea 25: 141. Accidentally omitted from latest checklist (Hackman 1980 Notulae Entomol. 60: 17-48). Anaretella defecta (Winn.) Ab: Uusikaupunki. Økland \& Mamaev 1997 Fauna Norv., Ser. B 44: 125 .

- magnicornis Mam. Ab: Uusikaupunki. ibid.: 125.

Bryomyia apsectra Ab: Uusikaupunki. Økland \& Mamaev 1997: 126. Mamaev \& Økland 1998 EF 9: 151.

Colobostema infumatum (Halid.) N: Tuusula (R. Tuomikoski); "Lapponia" (E. Palmén). Krivosheina 2000 Entomol. Obozr. 79: 491.

Bibio varipes Meig. Al: Lemland (H. Silfverberg). Skartveit 1999 EF 10: 115.

- lepidus Loew (sp. dist.) N: Helsinge (C. Lundström). ibid.: 115.

Dilophus borealis Skartv. Al, St, Ta, Sa, Om, Ok, $O b, K s$. ibid.: 115.

Scenopinus vitripennis Meig. Oa: Isokyrö. Haarto 2000 EF 11: 1.

Hilara hybrida Coll. Ob, Lk, Le, Li. Chvála 1996 System. Entomol. 21: 274.

- femorella Zett. Le: Kilpisjärvi. Frey 1930 Notulae Entomol. 10: 113; Chvála 1996: 280. Accidentally omitted from latest checklist (Hackman 1980 Notulae Entomol. 60: 17-48).

- submaura Coll. Ks: Kuusamo (R. Frey), Le: Enontekis (E. Palmén). Chvála 1996: 286.

Clinocera aucta (Zett.) (sp.dist.) $K s, L e, L i$. Sinclair 1999 Ent. Mon. mag. 135: 229.

Sciapus contristans (Wied.) Ab: Dragsfjärd Örö (Clayhills, Koponen \& Rinne). Haarto 1997 EF 8: 1.

Aenigmatias lubbocki (Verr.) Ab: Mietoinen. Haarto 1998 EF 9: 1.

Platycheirus complicatus Beck. Li: Utsjoki. Haarto 1995 EF 6: 5.

- amplus Curr. Ab: Nousiainen (T. Lammes). Haarto 1997 EF 8: 1.

- nielseni Vocker. Al: Eckerö, Ta: Somero, Li: Utsjoki. Haarto 1997: 1. Bartsch 2000 Sahlbergia 5: 45 .

- occultus Goeld., M. \& Sp. Ab, Ta. Haarto 1997: 2. Haarto 1998 EF 9: 2. Mattila 2000 Diamina 9: 44.

- holarcticus Vock. Finland. van Steenis \& Goeldin 1998 Mitt. Schweiz. Ent. Ges. 71: 193. Mattila 2000: 44. 
- nigrofemoratus Kan. Le: Kilpisjärvi. Haarto 2000 EF 11: 1.

Dasysyrphus hilaris (Zett.) Al: Eckerö. Bartsch 2000

Epistrophella euchroma (Kow.) Ab, Ta. Haarto 1997 EF 8: 2.

Epistrophe cryptica Dozc. \& Schm. Ab: Nousiainen (T. Lammes). Haarto 1997: 2.

- eligans (Harr.) Al: Eckerö \& Jomala. Bartsch 2000: 44.

Eupeodes abiskoensis (Dušek \& Láska) Le: Kilpisjärvi, Li: Utsjoki. Haarto 1995: 5 . Haarto 1997: 2.

- latilunulatus (Coll.) Sa: Savonlinna. Haarto 1997: 2.

— borealis (Duš. \& Lás.) Li: Utsjoki (S. Koponen $\&$ E. Linnaluoto). ibid.: 2.

Sphaerophoria virgata Goeld. Al, Ab, Ob, Ks. Haarto 1995: 6. Haarto 1996 EF 7: 2. Bartsch 2000: 45.

Syrphus admirandus Goeld. Ab, N, Ta. Haarto 1999 EF 10: 1.

Cheilosia sootryeni Niels. Ta: Lempäälä (J. Salokannel). Haarto 2000 EF 11: 1; Haarto 2000 Diamina 9: 7.

Eristalinus aeneus (Scop.) Ab: Korpo Jurmo (K. Rainio). Haarto 1999: 1.

Eristalis gomojunovae Viol. Lk, Le, Li. Nielsen 1995 Intern. J. Dipterol. Res. 6: 132.

Anasimyia interpuncta (Harr.) Al: Eckerö. Bartsch 2000: 44.

Pipizella viduata (L.) Al, Ob. Haarto 1995: 5. Bartsch 2000: 45.

* Palloptera anderssoni Roth. \& McG. "near Lempola" (Chandler). Rotheray \& MacGowan 1999 Brit. Journ. Entomol. Nat. Hist. 11: 175.

Stiphrosoma laetum (Meig.) (cingulatum auct.) Finland. Roháček 1996 Europ. J. Entomol. 93: 119.

Chlorops centromaculatus (Duda) N: Helsinki (M. Koponen), Ta: Kärkölä (M. Koponen). Nartshuk 1998 EF 9: 167.

- crassipalpis Smirn. N: Helsinki (C. Ahnger). ibid.: 167.

- rossicus Smirn. Ab, St, Tb, Kb, Li. ibid.: 170.

- serenus Loew Al: Lemland (Lindberg), Kökar (Koponen). ibid.: 172.

- zonulatus Wahlgr. Al, Ab, N, Ta, Kl, Sb, Ks. ibid.: 173.

Cryptonevraflavitarsis (Meig.) Al-Om (accidentally omitted from checklist in 1980). Nartshuk
1999 EF 10: 14.

Cetema simile Ism. $\mathrm{A} b, \mathrm{~N}, \mathrm{Ta}, \mathrm{Sb}, \mathrm{Om}$. Nartshuk 1999 EF 10: 15.

- neclectum Tonn. Al, Ab, N, Sa. ibid.: 15.

Eutropha fulvifrons (Halid.) N: Hangö (R. Krogerus). ibid.: 16.

Pseudopachychaeta heleocharis (Nartsh.) Ta,Om. ibid.: 17

- ruficeps (Zett.) Ab, N, Ta, Sa, Oa, Tb, Sb, Om, $O k, O b, K s, L k, L e, L i$. ibid.: 17.

Lasiosina albipila (Loew) N: Helsinki. ibid.: 17.

- brevisurstylata Dely-Dr. St: Eura. ibid.: 17.

Amobia signata (Meig.) Oa: Ilmajoki. Haarto 2000 EF 11: 1.

Chaetostevenia brevicornis (Zett.) Ab: Rymättylä. ibid.: 1.

Pseudoperichaeta nigrolineata (Walk.) Ab: Kaarina (V.-M. Mukkala). Haarto 1999: 1.

Lydella ripae Brisch. Dragsfjärd Örö (Clayhills, Koponen \& Rinne). Haarto 1997 EF 8: 2.

Eryciafatua (Meig.) Sa: Joutseno. Komonen 1997 Baptria 22: 108.

Peleteria [Cuphocera] ruficornis Macq. Ab: Naantali (I. E. Sääksjärvi). Haarto 1999: 1.

Ceromya flaviceps (Ratz.) Ob: Pisavaara (H. Lindberg). Andersen 1996 Fauna Ent. Scand. 33: 43.

Entomophaga sufferta Vill. N: Inkoo (Koponen). ibid.: 54.

Actia maksymovi Mesn. Ab: Nagu (A. Nordman). ibid.: 61

*Peribaea hertingi And. Ab: Lojo (Forsius), $N$ : Hangö (Hellén), ibid.: 74.

Siphona ingerae And. Al, N, Ka, Ta. ibid.: 86.

*_immaculata And. Al: Saltvik (M. Meinander). ibid.: 89.

- collini Mesn. Al, Ab, N. ibid.: 90.

- confusa Mesn. Al, N, Ks. ibid.: 92.

- urbana (Harr.) Al-Ks. ibid.: 97.

- setosa Mesn. Ab, N, Ka, Ta, Sa, Ks. ibid.: 99.

- pauciseta Rond. Ab: Nagu (M. Meinander). ibid.: 100.

*_ subarctica And. Sb, Ks, Lk, Li. ibid.: 103.

- rossica Mesn. Al, N. ibid.: 106.

Aphantorhaphopsis samarensis (Vill.) Ta: Kuhmoinen, Harmoinen (E. Hellman), Kl: Parikkala (W. Hellén). ibid.: 110.

Ceranthia lichtwardtiana (Vill.) N, Ta, Sa, Kb. ibid.: 120.

*_verneri And. Ab, N, Ka. ibid.: 121. 
— pallida Hert. Ta: Janakkala(Peltonen). ibid.: 122.

- tenuipalpis (Vill.) N: Tvärminne (A. Nordman). ibid.: 122.

Aphria longilingua Rond. Ab: Dragsfjärd Örö. Haarto 1999: 1.

Ramonda ringdahli (Villen.) Li: Utsjoki. ibid.: 1 .

Present number: $5237-17+129=5349$ spp.

\section{Hymenoptera}

Koponen, M., Jussila, R. \& Vikberg, V. 1999. Suomen loispistiäisluettelo (Hymenoptera, Parasitica). Osa 2. heimo Ichneumonidae, alaheimot Tryphoninae, Eucerotinae, Adelognathinae, Xoridinae ja Agriotypinae. Sahlbergia 4: 1-18.

Koponen, M., Jussila, R. \& Vikberg, V. 1999. Suomen loispistiäisluettelo (Hymenoptera, Parasitica). Osa 3. heimo Ichneumonidae, alaheimo Cryptinae. - Sahlbergia 4: 19-52.

Koponen, M., Jussila, R. \& Vikberg, V. 2000. Suomen loispistiäisluettelo (Hymenoptera, Parasitica). Osa 4. heimo Ichneumonidae, alaheimot Lycorininae, Neorhacodinae, Stilbopinae, Banchinae ja Ctenopelmatinae. — Sahlbergia 5: 51-82.

\section{Deletions}

Amauronematus stenogaster Först. (= A. fallax (Serv.)). Schmidt 1997 Beitr. Entomol. 47: 280.

- festivus Saar. (= A. fallax (Serv.)). ibid.: 280.

Thymaris pulchricornis Brischke $(=T$. tener (Grav.)). Koponen et al. 1999: 5.

- collaris (= T. tener (Grav.)). ibid.: 5.

Polyblastus carbonarius (Grav.) (f.d. = P. subalpinus Holmgr.). ibid.: 7.

Ctenochira pratensis (Grav.) (f.d. = C. pectinata (Bauer)), ibid.: 8.

Exyston phaeorrhoeus (Hal.) (f.d. = E. pratorum (Woldst.)). ibid.: 10.

Hemiteles unicolor Thoms. (= H. similis (Gmel.)). Koponen et al. 1999: 25.

Polyaulon rufipes Först. (f.d. = P. paradoxus (Zett.)). Horstmann 1998 Entomofauna 19: 454. Koponen et al. 1999: 23.

Gelis edentatus (Först.). Recorded outside Finland. Koponen et al. 1999: 23.

- linearis (Först.) (= G. anthracinus (Först.)). ibid.: 27.

Arctodeuon glacialis (Thoms.) (f.d. = Gelis alopecosae Horstm.). ibid.: 20.

Thaumatogelis aquisgranensis (Först.) (= T. vulpinus (Först.)). ibid.: 28.

Endasys kriegeri (Haberm.) (= E. cnemargus (Grav.)), ibid.: 29.

Charitopes macrocerus Hellén. Recorded outside Finland. ibid.: 23.

Isadelphus obscuripes (Thoms.) (= I. inimicus (Grav.)). ibid.: 31.

Bathythrix rubens (Kriechb.) (= B. pellucidator (Grav.)). ibid.: 32.

Leptocryptoides clavipes (Thoms.). Recorded outside Finland. ibid.: 23.

Stibeutes liogaster (Thoms.) (= S. infernalis (Ruthe)), ibid.: 33.

Phygadeuon fasciatus Brischke (= Orthizema hadrocera (Thoms.)). ibid.: 33.

- lateareolatus Hellén. Recorded outside Finland. ibid.: 23.

- punctigena Thoms. (= P. leucostigmus Grav.). ibid.: 35.

- rugipectus (Thoms.) (=P.pallicarpus (Thoms.)). ibid.: 35.

- striiventris Hellén. Recorded outside Finland. ibid.: 23.

Parmortha erythropus (Grav.) (= P. parvula (Grav.)). ibid.: 38.

Gambrus inferus Thoms. (= G. incubitor (L.)). Schwarz \& Shaw 1998 Entomol. Gaz. 49: 111.

- ornatulus Thoms. (=G.incubitor (L.)). ibid.: 111 .

Enclisis remex (Tschek) (=E. macilentus (Grav.)). Koponen et al. 1999: 43.

- nubifer (Thoms.) (=E. vindex (Tschek)). ibid.: 43.

Caenocryptus melanarius Hellén (= C. polytomi (Tschek). ibid.: 44.

Schreineria annulata (Brischke). Recorded outside Finland. ibid.: 23.

Stilbops ruficornis (Grav.). Recorded outside Finland. Koponen et al. 2000: 57.

Glypta nigrina Desv. Recorded outside Finland. ibid.: 57.

- rufipes Brischke (= G. nigricornis (Thoms.)). Koponen et al. 2000: 58.

Lissonota perspicillator Grav. Recorded outside Finland. ibid.: 57.

- unicincta Holmgr. (= L. rusticator (Thunb.). ibid.: 61 .

- segmentator auct. nec (F.) (= L. rusticator (Thunb.). ibid.: 61. 
Rhorus haemorrhoicus (Hart.) (= R. substitutor (Thunb.)). ibid.: 65.

Rhaestus femoralis auct. nec Thoms. (= R. lativentris (Holmgr.). ibid.: 65.

Perilissus holmgreni Haberm. (= P. pallidus (Grav.)). ibid.: 66.

Rhinotorus atratus (Holmgr.) (= R. compactor (Thunb.)). ibid.: 69.

Notopygus emarginatus Holmgr. Recorded outside Finland. ibid.: 57.

- xanthocerus Kriechb. Recorded outside Finland. ibid.: 57.

Rhinotorus forsii (Rom.) (=Campodorus assiduus (Holmgr.)). Kasparyan 1997 Zoosyst. Ross. 6: 302 .

Campodorus alni (Woldst.) (= C. haematodes (Grav.)). Koponen et al. 2000: 70.

- drewseni (Holmgr.) (= Mesoleius dubius Holmgr.). Kasparyan 1997: 302.

- nubilis (Holmgr.). Recorded outside Finland. Koponen et al. 2000: 57.

- subfasciatus (Holmgr.). Recorded outside Finland. ibid.: 57.

Mesoleius curvicrus Holmgr. (= M. grossulariae (Ratz.)). Kasparyan 2000 Entomol. Obozr. 79: 163.

- discedens Holmgr. (= M. grossulariae (Ratz.)). Kasparyan 2000: 163.

- erythrogaster Holmgr. (=M. axillaris (Steph.). Kasparyan 2000: 153.

- tenuiventris Holmgr. (= M. axillaris (Steph.). Kasparyan 2000: 153.

- wahlbergi Holmgr. (= M. grossulariae (Ratz.)). Kasparyan 2000: 163.

Saotis brevispina (Thoms.). Recorded outside Finland. Koponen et al. 2000: 57.

Mesoleptidea sylvatica (Woldst.). Recorded outside Finland. ibid.: 57.

Hypamblys transfugus (Holmgr.) (=H. albopictus (Grav.)). ibid.: 75.

Phobetes latipes (Thoms.) (= P. nigriceps (Grav.)). ibid.: 76.

Campoletis holmgreni (Tschek) (= C. ensator (Grav.)). Horstmann 2000 Linz. Biol. Beitr. 32: 1206.

- erythropus (Thoms.) (=C. rapax (Grav.)). ibid.: 1208.

Probles pallipes (Holmgr.) (= P. gilvipes (Grav.)). Horstmann 1971 Veröff. Zool. Staatssamml. München 15: 90.
Stictopisthus complanatus (Halid.) (= S. unicinctor (Thunb.)). Schwenke 1999 Spixiana Suppl. 26: 31.

Mesochorus nigriceps Thoms. nec Brischke (=M. punctipleuris Thoms.). ibid.: 44.

— tachypus Holmgr. (= M. gemellus Holmgr.). ibid.: 108.

Triaspis arcticus Hellén (= T. caudatus (Nees)). Papp 1999 Folia Entomol. Hungar. 60: 284.

Eubazus atricornis (Ratz.) (= E. semirugosus (Nees). van Achterberg \& Kenis 2000 Zool. Meded. 73: 448.

- mucronatus (Thoms.) (= E. semirugosus (Nees). ibid.: 449.

Microgaster grandis Thoms. (= M. meridianus Halid.). van Achterberg 1997 Zool. Verhand. 317: 58.

Apanteles memnon Nix. (= Cotesia praepotens (Halid.)). ibid.: 68.

Opius exilis Halid. (=O.pallipes Wesm.). ibid.: 38.

Psyttalia carinata (Thoms.) (f.d. = P. rhagoleticola (Sachtl.)). Fischer \& Koponen 1999 EF 10: 144.

Biosteres sylvaticus (Halid.) (f.d.). ibid.: 149.

Torymus rosarum (Hoffm.) (=T. bedeguaris (L.)). de Vere Graham \& Gijswijt 1998 Zool. Verhand. 317: 64.

- glechomae Mayr (=T. cingulatus Nees). ibid.: 76.

- tiliarum Ruschka (= T. eglanteriae Mayr). ibid.: 90.

Codrus buccatus (Thoms.) (= Parthenocodrus elongatus (Halid.). Johnson 1992 Mem. Amer. Ent. Inst. 51: 313.

- gracilis (Nixon) (= Exallonyx ater (Grav.) (aterrimus (DT)), ibid.: 291.

Zygota longinervis (Kieff.) (= Synbelyta fuscipennis Thoms.) Macek 1996 Čas. Nár. Muz., rad. prír. 165: 67.

Belyta brevicornis (Kieff.) (f.d. = B. borealis Whitt.) Macek 1996 Sborn. Nár. Muz. Praze, Ser. B 51: 20.

- brachyptera Thoms. (= B. sanguinolenta Nees) ibid.: 10.

Trimorus angustulus (Thoms.) (= T. flavipes (Walk.)). Johnson 1992: 532.

- autumnalis (Thoms.) (=T. therycides (Walk.). ibid.: 552.

- pallipes (Thoms.) (= T. therycides (Walk.). ibid.: 552. 
- medon (Walk.) (=Xenomerus ergenna Walk.). ibid.: 559

Spilomena expectata Valk. (= S. beata Blüthg.). Vikberg 2000 EF 11: 41.

\section{Unjustified deletion}

Gelis helleni Kolar. (breviceps sensu Hellén). Deleted as recorded outside Finland, but also reported from $N$ : Helsinge. Hellén 1970 Notulae Entomol. 50: 86. Koponen et al. 1999: 23.

\section{Addition later deleted}

Opius insertus Fisch. "Finnland" (sp. dist.) Fischer 1997 Entomofauna 18: 139. (= O. zelotes Marsh.) Fischer \& Koponen 1999 EF 10: 77.

\section{Additions}

*Euura cinereae Kop. Kb: Joensuu (Roininen). Kopelke 1996 Senckenbergiana biol. 76: 102.

- weiffenbachii Ermol. Ab Kaarina (Roininen). ibid.: 109.

Parna reseri List. $N$ : Borgå (Hellén), Ta: Pälkäne (J. Kangas). Heidemaa \& Viitasaari 1997 Proc. Eston. Acad. Sci. Biol. Ecol. 46: 192.

*Caliroa crypta Heid. Ab: Perniö (P. Niemelä). Heidemaa 1999 EF 10: 183.

*Amauronematus betulae Schm. Ks: Kuusamo (M. Ahola). Schmidt 1997 Beitr. Entomol. 47: 279.

*_mimus Schm. Ta: Pälkäne (J. Kangas). ibid.: 285.

*_nigrinus Schm. Finland. ibid.: 286.

*_subfuscus Schm. Tb: Toivakka (E. Tiihonen). ibid.: 294.

*_tenuis Schm. Li: Utsjoki Kevo. ibid.: 295.

Scambus eucosmidarum (Perk.) Ab, St. Jussila 1999b EF 10: 175.

Phytodietus crassitarsis Thoms. (sp.dist.). Koponen et al. 1999: 4.

- segmentator Grav. (sp.dist.). ibid.: 4.

Eclytus rubridorsum Kasp. Ob: Pello (R. Hinz). Kasparyan 1977 Ent. Obozr. 56: 168. Koponen et al. 1999: 5.

Thymaris niger (Tasch.) Al, Ab, N, Ob. Koponen et al. 1999: 1.
— srikem Fitt. \& Fick. Ab, N, Sa. ibid.: 1.

- tener (Grav.) (pulchricornis Brischke, collaris (Thoms.)). Finland. ibid. 5.

Cladeutes discedens (Woldst.) Al: Föglö (R. Jussila), $A b$ : Vihti (A. Petramaa), ibid.: 2.

Cosmoconus hinzi Kasp. Ab: Vihti, $N$ : Kirkkonummi. ibid.: 2.

Tryphon brevipetiolaris Uch. Ab, N, Ta. ibid.: 2.

- fulviventris. Holmgr. Ta: Forssa, Sb: Leppävirta (E. Tiihonen). Jussila 1999c Loun. Häm. Lu 86: 25. Koponen et al. 1999: 3.

- hinzi Heinr. Ab, N, Ta, Sa, Sb, Li. Koponen et al. 1999: 2.

Grypocentrus bilobus Kasp. N: Helsingfors (A. Nordman), ibid.: 2.

Polyblastus alternans Schte. Ab, N, Ta. ibid.: 2. Jussila 1999c:25.

- leucoon Kasp. Ab, N, Ka,Ta. ibid.: 2.

Ctenochira annulata (Holmgr.) Ab, Ka, Ta, Sa, $O k$. ibid.: 2.

— bisinuata Först. Le: Kilpisjärvi (V. Karvonen). ibid.: 2.

- fecula Kasp. N: Kirkkonummi (V. Karvonen) \& Espoo (J. Perkiömäki). ibid.: 2.

— gelida Kasp. Le Kilpisjärvi (V. Karvonen), Li: Utsjoki (V. Karvonen). ibid.: 2.

- genalis (Thoms.) Ab, N, Ka, Ta, Le ibid.: 2.

- oreophila (Schmied.) Ab: Vihti (V. Karvonen). ibid.: 3 .

- sculpturata Kasp. Le: Kilpisjärvi (V. Karvonen), $L i$ : Utsjoki (V. Karvonen). ibid.: 3.

- xanthopyga (Holmgr.) Ab, N, St, Ta. ibid.: 3.

Erromenus alpestrator Aub. $N$ : Kirkkonummi (V. Karvonen), ibid.: 3.

- tarsator Aub. Ab, N, Sb, Li. ibid.: 3.

Orthomiscus unicinctus (Holmgr.) Ab: Vihti (V. Karvonen), Ta: Kangasala (Th. Grönblom). ibid.: 3 .

!Dyspetes praerogator (Thoms.) $N$ : Espoo, Ka: Virolahti. Jussila 2000 EF 11: 88.

Adelognathus cubiceps Rom. Le: Enontekiö. Kopelke 1994 Senckenbergiana biol. 73: 102. Koponen et al. 1999: 4.

- nigriceps Thoms. Ab: Vihti (V. Karvonen \& A. Petramaa). ibid.: 4.

Xorides gracilicornis (Grav.) Ab: Turku (R. Lindqvist). ibid.: 4.

!- rufipes (Grav.) Finland. Hilszczanski 2000 Ins. Syst. Evol. 31: 254.

Pleurogyrus cyclogaster (Thoms.) St: Huittinen 
(R. Jussila), Ta: Urjala (T. Reilin). Koponen et al. 1999: 19. Jussila 1999c:27.

Aclastus eugracilis Horstm. Ab: Korppoo (R. Jussila). Koponen et al. 1999: 19.

- flavipes Horstm. Al: Lumparland (R. Jussila). ibid.: 19.

Polyaulon stiavnicensis Čap. N: Hanko (M. Koponen), ibid.: 19.

Dichrogaster genalis (Haberm.) Ab, N, St. ibid.: 19.

Gelis alopecosae Horstm. Ok: Suomussalmi (O. Sorsakoski), Li: Utsjoki (W. Hellén). ibid.: 20.

- bicolor (Vill.) Al, Ab, St, Ta, Oa. ibid.: 20.

- discendens (Först.) Ab, St. ibid.: 20.

- longulus (Zett.) Ab, N, Oa, Kb, Om. ibid.: 20.

- nigritulus Zett. Sa: Joutseno. Komonen 1997 Baptria 22: 108.

- thomsoni (Schmied.) Ab: Turku \& Sauvo. Koponen et al. 1999: 20.

Endasys annulatus (Haberm.) Ab: Sauvo, Ta: Urjala \& Forssa. Jussila 1999b:176. Jussila 1999c:28.

- bicolor (Lundb.) Ab, N, Tb, Le, Li. Koponen et al. 1999: 20.

- eurycerus (Thoms.) N: Helsinki (V. Karvonen), St: Huittinen (R. Jussila). ibid.: 20.

- testaceipes (Brischke) Ab, Tb. ibid.: 20.

Charitopes brunneus (Morl.) Ab: Turku, Oa: Vaasa. ibid.: 20.

Mastrus pictipes (Grav.) Ab: Turku. Jussila 1999b:175.

— tenuicosta (Thoms.) Ab: Turku, Tb: Saarijärvi. ibid.: 175.

Sulcarius hellbachi (Schmied.) (sp. dist.) Ab, Ta, Sa, Oa, Tb, Sb, Kb. Koponen et al. 1999: 23, 33.

Orthizema eleganticornis (Schmied.) Ab: Vihti (V. Karvonen), ibid.: 20.

- subannulata (Bridgm.) Ab, N, Ka, Ta, Tb, Sb, $O b$, Li. ibid.: 20.

Theroscopus trochanteratus (Thoms.) Ab, St. ibid.: 21.

Phygadeuon alpinus (Thoms.) Le, Li. ibid.: 21.

- brevitarsis Thoms. Ab, N, Ka, Sa. ibid.: 21.

- crassicornis (Grav.) N: Helsinki (V. Karvonen), ibid.: 21.

- occisor Haberm. Ab: Turku. Jussila 1999b:176.

- punctiventris Thoms. Ab, N, Oa, le, Li. Koponen et al. 1999: 21.

- stilpninus Thoms. Al, Ab, N, Ka, Ta, Oa, Le, Li. ibid.: 21.

!Stilpnus fallax (Först.) Al, Sb, Li. Jussila 1999a
EF 10: 107.

Mesoleptus filicornis (Thoms.) N: Helsinki (M. Koponen). Koponen et al. 1999: 21.

- marginatus (Thoms.) Ab, N, Ka, Sa, Li. ibid.: 21.

Atractodes townesi Juss. Al: Föglö (R. Jussila). ibid.: 22.

!— romani Juss. $T b$ : Keuruu (Y. Ermala). Jussila 1999b:176.

Plectocryptus digitatus (Gmel.) Ab, N, Ta. Koponen et al. 1999: 22.

Aptesis nigritula (Thoms.) Ab, Ta, Oa, Li. ibid.: 22. Aritranis explorator (Tschek) Ab, Ob. ibid.: 22.

Idiolispa obfuscator (Vill.) Li: Inari (V. Karvonen), ibid.: 23.

Trychosis gradarius (Tschek) Ab: Vihti (V. Karvonen), ibid.: 22.

Enclisis ornaticeps (Thoms.) Ab, Ta, Li. ibid.: 22.

Cryptus obscuripes Zett. (sp. dist.). Finland. ibid.: 44.

Stenarella domator (Poda) (gladiator (Scop.)) Ab: Turku \& Naantali. Sääksjärvi 1998 EF 9: 8; Koponen et al. 1999: 45.

Glypta salicis Thoms. Finland. Koponen et al. 2000: 58.

— similis Bridgm. Finland. ibid.: 59.

Lissonota cruentator (Panz.) (insignita (Grav.)). Finland. ibid.: 62.

- digestor (Thunb.) Finland. ibid.: 60.

— excelsa Schmied. $A b$ : Vihti, $N$ : Tvärminne \& Vantaa. ibid.: 51.

- flavovariegata (Luc.) (nigricoxis Strobl) (sp. dist.) Finland. ibid.: 62.

— nigridens Thoms. Finland. ibid.: 61.

- rusticator (Thunb.) (unicincta Holmgr., segmentator auct. nec (F.)). Finland. ibid.: 61.

Alloplasta plantaria (Grav.). Finland. ibid.: 62.

Syzeuctus [Cryptopimpla] helveticus Brauns Kb: Ilomantsi. Humala 1995 Karel. Biosph. Res. Stud., p. 157.

Xenoschesis mordax (Thoms.) (sp. dist.). Finland. Koponen et al. 2000: 64.

Homaspis [Notopygus] flavicornis Holmgr. Ta: Tammela. Jussila 1999c: 32.

- varicolor (Thoms.) Finland. Koponen et al. 2000: 64.

Trematopygus rufator Hinz Li: Utsjoki (R. Jussila). ibid.: 52 .

- spiniger Hinz $K b$ : Hammaslahti (P. Kontkanen). ibid.: 52. 
Lethades cingulator Hinz Ab: Sauvo (R. Jussila). ibid.: 51.

- curvispina (Thoms.) Ab, N, Ta, Oa, Tb, Ks, Le, Li. ibid.: 51.

Rhorus chrysopygus (Roman) Li: Utsjoki (K. Ruohomäki). ibid.: 52.

Sympherta splendens (Strobl) Ab, Tb, Sb. ibid.: 52.

Perilissus lutescens Holmgr. N, Ta, Kl. Hellén 1961 Notulae Entomol. 41: 94. Jussila 1999c: 33.

Lathrolestes lucidulus (Holmgr.) Ab: Vihti (V. Karvonen), $N$ : Kirkkonummi (V. Karvonen). Koponen et al. 2000: 52.

Scolobates fennicus Schmied. (sp. dist.). Finland. ibid.: 67.

Alexeter coxalis (Brischke) Al, Ab, N, Ka, Tb, Kb, $O b, L i$. ibid.: 52.

Lamachus transiens (Ratz.) Finland. ibid.: 69.

Rhinotorus mesocastaneus (Thoms.) Ks, Le, Li. ibid.: 52.

Campodorus astutus (Holmgr.) N: Helsinki (I. Jalas). ibid.: 52.

- axillaris (Steph.) Ab, Oa, Tb, Sb, Ob, Ks, Le, Li. ibid.: 52.

- ciliatus (Holmgr.) N: Tvärminne (V. Karvonen). ibid.: 52.

- commotus (Holmgr.) Al, Ab, N, Ka, Tb, Lk. ibid.: 52.

- crassipes (Thoms.) N: Kirkkonummi (V. Karvonen), ibid.: 53.

- crassitarsis (Thoms.) Finland. ibid.: 70.

- holmgreni (Schmied.) N: Kirkkonummi \& Vantaa. ibid.: 53.

- humerellus (Thoms.) Ab, N, Tb. ibid.: 53.

- intermedius (Grav.) Ab, N, Le. ibid.: 53.

- latiscapus (Thoms.) Sa: Hirvensalmi (O. Ranin). ibid.: 53.

- nigridens (Thoms.) Ab, N, St, Om. ibid.: 53.

- vestergreni (Roman) Le: Kilpisjärvi (V. Karvonen), ibid.: 53.

— vicinus (Holmgr.) Le: Kilpisjärvi (V. Karvonen). ibid.: 53.

Mesoleius affinis Brischke Tb: Toivakka (E. Tiihonen), ibid.: 53.

* - antennator Kasp. Le: Kilpisjärvi (Hinz). Kasparyan 2000 Entomol. Obozr. 79: 164.

- axillaris (Steph.) (amabilis Holmgr., erythrogaster Holmgr., tenuiventris Holmgr.) [Finland]. Kasparyan 1997 Zoosyst. Ross. 6: 302. Kasparyan 2000: 153.

- cognatus Brischke Ab: Vihti (V. Karvonen), $N$ :
Helsinki (I. Jalas). Koponen et al. 2000: 53.

- contractus (Holmgr.) Finland. ibid.: 71.

- grossulariae (Ratz.) (curvicrus Holmgr., discedens Holmgr., wahlbergi Holmgr.) [Finland]. Kasparyan 1997: 302. Kasparyan 2000: 163.

- parvus Holmgr. Ab: Lieto (R. Jussila), Le: Kilpisjärvi (V. Karvonen). Koponen et al. 2000: 53.

- placidus Holmgr. Ab, N, Ob. ibid.: 53.

- subcoriaceus Strobl Ab: Vihti (V. Karvonen), $N$ : Kirkkonummi (V. Karvonen). ibid.: 53.

!*_tibiator Kasp. Le: Kilpisjärvi (Hinz). Kasparyan 2000: 176.

- varicoxa Thoms. Ab, N, Ok, Le. Koponen et al. 2000: 53.

Hyperbatus aemulus (Holmgr.) Ab, N, Le. ibid.: 53.

Saotis compressiventris (Strobl) Ab, N, Sb. ibid.: 54 .

- longiventris (Thoms.) Ab: Vihti, $N$ : Kirkkonummi (V. Karvonen), ibid.: 54.

Anoncus flavicaudatus (Holmgr.) Le: Kilpisjärvi (V. Karvonen). ibid.: 54.

- sincerus (Holmgr.) Ab: Turku (R. Jussila), Le: Kilpisjärvi (V. Karvonen), ibid.: 54.

Synomelix faciator Idar $A b, N, S b, L k, L e$. ibid.: 54 .

Hadrodactylus bidentulus Thoms. Al, Ab, N, St, $\mathrm{Sa}, \mathrm{Tb}, \mathrm{Sb}, \mathrm{Ob}, \mathrm{Li}$. ibid.: 54.

- graminicola Idar $A b, N, S t, T b, K b, K s, L i$. ibid.: 54.

— indefessus (Grav.) Ta (m.1.). Jussila 1999c:35.

- rectinervis Roman Ab: Vihti (V. Karvonen), $L i$ : Utsjoki (R. Jussila). Koponen et al. 2000: 54.

- spiraculator Idar $A b, N, S a$. ibid.: 54.

- villosulus Thoms. Ab, N, Sb, Ob. ibid.: 54.

Syndipnus abbreviatus Roman N: Vantaa (O. Ranin). ibid.: 55.

- albicruris (Grav.) Ab, Sb. ibid.: 55.

- decipiens Woldst. Le: Enontekiö (R. Frey), Li: Utsjoki (W. Hackman). ibid.: 55.

- flaviventris Roman Ab: Sauvo (R. Jussila), Le: Enontekiö (E. Karvonen), ibid.: 55.

Phobetes femorator (Thoms.) Ta: Tammela. Jussila 1999c: 35.

- rufipes (Thoms.) Al, Ab, N,Ta. Koponen et al. 2000: 55.

- splendidissimus (Strobl) Ab, N, Tb, Sb, Kb. ibid.: 55.

Euryproctus inferus Thoms. Ab: Vihti (V. Karvonen), $N$ : Helsinki (O. Ranin). ibid.: 55.

- plantator (Thunb.) (tuberculatus Holmgr.) (sp. 
dist.) Finland. ibid.: 76.

Delopia polita (Först.) Ta: Urjala. Jussila 1999c: 36.

*Lathroplex anthreni Vikb. Ta, Sa, Kb. Vikberg 1999 EF 10: 239.

- clypearis Thoms. N: Nurmijärvi (M. Koponen), ibid.: 242.

Leptocampoplex cremastoides (Holmgr.) Ab: Korpo (M. Nieminen). Várkonyi 1998 EF 9: 215.

Diadegma claripennis (Thoms.) Kb: Ilomantsi. Humala 1995: 157.

Lathrostizus flexicauda (Holmgr.) Le: Kilpisjärvi. Kopelke 1994: 99.

*Astrenis nigrifacies Vikb. Ab, N, St, Ta, Oa, Tb, $K b, O k$. Vikberg 2000 EF 11: 210.

*_brunneofacies Vikb. Ab, N, Ta, Sa, Oa, Tb, Ok. ibid.: 213.

Phrudus defectus Stelf. Ab, N, Ta, Sa, Tb, Ob, Lk, Li. ibid.: 218.

*_compressus Vikb. Ta: Janakkala \& Riihimäki. ibid.: 220.

— badensis Hilp. Ta, Sa, Kl. ibid.: 221.

Diaparsis frontellus (Holmgr.). Finland. Horstmann 1971 Veröff. Zool. Staatssamml. München 15: 99.

Epistathmus crassicornis Horstm. Finland. ibid.: 108.

Tersilochus spiracularis Horstm. Finland. ibid.: 125.

* Mesochorus boreomontanus Schw. "Oshobotria" (R. Müller). Schwenke 1999 Spixiana Suppl. 26: 86.

*_ suomiensis Schw. "Oshobotria bor." (K. Müller). ibid.: 91.

*_fennicus Schw. Li: Utsjoki (Rinohomäki). ibid.: 94

Chorinaeus flavipes Bridgm. Ab: Turku. Jussila 1996 EF 7: 97.

Trieces dinianae Aeschl. Sb: Varpaisjärvi, Ok: Kuhmo. ibid.: 98.

Metopius harpyiae Clém. Ob: Tornio (J. Itämies), Ks: Oulanka (J. Itämies). Várkonyi 1998 EF 9: 52.

Tersilochus spiracularis Horstm. Ta: Forssa. Jussila 1999c: 40.

Exochus lentipes Grav. Ta: Tammela \& Urjala. ibid.: 41.

Pantisarthrus dispar Ross. Kb: Ilomantsi. Humala 1995: 157.

Proeliator proprius Ross. Kb: Ilomantsi. ibid.: 157. Proclitus ardentis Ross. Kb: Ilomantsi. ibid.: 157.

Eusterinx circaea Ross. Al: Saltvik (W. Hellén),
Ab: Dragsfjärd (W. Hellén). Humala 1997 Zoosyst. Ross. 5: 300.

Helictes conspicuus (Först.) Kb: Ilomantsi. Humala 1995: 157.

Syrphoctonus megaspis (Thoms.) Al, Ab, St. Jussila 1996: 95.

- collinus (Stelf.) Ta: Forssa. Jussila 1999c: 42.

Woldstedtius holarcticus (Dill.) Al, Ab, Ka, St, Ta, Oa. Jussila 1996: 95.

Diplazon pallicoxa Manuk. Ab: Sauvo. ibid.: 97.

Phaeogenes curator (Thunb.) Ta: Forssa \& Tammela. Jussila 1999c: 43.

Cotesia melitaearum (Wilk.) Al: Finström; Sa: Joutseno. Lei et al. 1997 Journ. Nat. Hist. 31: 637; Komonen 1997: 108.

*Microchelonus nigritus Tob. $N$ : Helsinki (M. Koponen). Tobias 1999 Zool. Zh. 78: 703.

— irritus Tob. N: Pernå (Nordström). ibid.: 707.

— incrassus Papp Ta: Ypäjä, Lammi, Koski \& Padasjoki (P. Peltotalo). Papp 1992 Ann. Hist. Nat. Mus. Natn. Hungar. 84: 134.

Opius decoratus Stelf. Ab: Nauvo, Turku. Fischer \& Koponen 1999 EF 10: 68.

- notabilis Fisch. Ab: Nauvo, Om: Reisjärvi. ibid.: 69.

— rudis Wesm. Sa: Imatra (Hk. Lindberg). ibid.: 69.

- holconotus Fisch. Ob: Simo, Li: Inari. ibid.: 69.

- karesuandensis Fisch. Ok: Paltamo, Lk: Muonio. ibid.: 71

— polyzonius Wesm. Ab, N, Ta, Sa,Tb. ibid.: 72.

- reconditor Wesm. Oa: Lappfjärd (Nordström). ibid.: 72.

- rufipes Wesm. Al, N, Sa. ibid.: 73.

— pactus Halid. N: Nurmijärvi. ibid.: 73.

- aemulus Halid. Al, N, Ta, Sa, Kb. Fischer 1997 Entomofauna 18: 139. Fischer \& Koponen 1999: 74.

- curtipectus Fisch. N: Nurmijärvi. Fischer \& Koponen 1999: 75.

- hilaris Fisch. Al, Ab, N, Ta, Sa, Kb, Om, Ob. ibid.: 76.

- magnus Fisch. $N$ : Helsinki. ibid.: 76.

— ochropus Thoms. N: Kirkkonummi. ibid.: 76.

- aureliae Fisch. Ab: Perniö, $N$ : Helsinki, Sa: Mikkeli. ibid.: 78 .

— flavigaster Fisch. Ab: Pojo (Meinander). ibid.: 78.

- crassicrus Thoms. Ab, N, St, Sa, Tb, Sb, Kb Ob. ibid.: 78 .

— tscheki Fisch. Ta: Janakkala. ibid.: 78.

- curvatus Fisch. Al, N, Ta, Sa. ibid.: 79. 
— gracilis Fisch. $N, T a, S a, K b, K s$. ibid.: 79.

- minor Fisch. Ab, N, St, Ta, Sa, Kl, Tb, Ks, Lk. ibid.: 79.

- pygmaeus Fisch. Al, Ab, N, St, Ta, Sa,Kl, Kb, $O k, O b, l k, L i$. ibid.: 80 .

- tersus (Först.) Al, Ab, N, Ta, Kl, Kb. ibid.: 80.

- caprifolii Fisch. Ta: Hattula. ibid.: 80.

- iuxtangelum Fisch. $N$ : Nurmijärvi. ibid.: 81.

- pulicariae Fisch. $N, T a, K b, K s$, Le ibid.: 81.

- austriacus Fisch. Ta: Lahti. ibid.: 81

— heringi Fisch. N, Ta, Sa. ibid.: 82.

- similis Szépl. N, Ka,St,Ta,Sa,Kb,Om ,Ks. Fischer 1997: 174. Fischer \& Koponen 1999: 83.

- similoides Fisch. Al, N, St, Ta, Sa, Kl, Kb, Om, $O k, O b, K s$. ibid.: 83.

- tirolensis Fisch. Ab, N, Ta, Sa, Ok. ibid.: 84.

- saevulus Fisch. $N$ : Siuntio. ibid.: 85.

- saevus Halid. N, Sa, Kb, Ob, Ks. ibid.: 85.

- mundus (Först.) $N$ : Nurmijärvi, Kl: Uukuniemi. ibid.: 85.

- celsus Halid. N: Sibbo, Ok: Kajaani. ibid.: 86.

- maculipes Wesm. N, Ta, Sa ibid.: 88.

— pirchitticola Fisch. Li: Inari. ibid.: 88.

- seebensteinensis Fisch. $N$ : Helsinki, Sb: Kuopio. ibid.: 88.

- sigmodus Papp Al: Mariehamn (W. Hellén). ibid.: 88.

- speciosus Fisch. N: Helsinki. ibid.: 88.

- tuberculifer Fisch. N, St, Ok, Ob. ibid.: 88.

— viennensis Fisch. Ta: Pälkäne (M. Hellén). ibid.: 89.

- connivens Thoms. N, Sa, Ob. ibid.: 129.

- epulatus Papp N: Helsinki (Yu). ibid.: 129.

— paranivens Fisch. N: Helsinki \& Nurmijärvi. ibid.: 130.

- parvungula Thoms. Ab, N, St, Sa. ibid.: 131.

- agromyzicola Fisch. $N$ : Nurmijärvi, Ks: Kuusamo. ibid.: 131.

- breviscapus Thoms. Al, Ab, N, St, Sa, Kl, Ok, Ob, Lk. ibid.: 132.

- caricivorae Fisch. N, Kb, Om. ibid.: 132.

- circinus Papp N: Nurmijärvi. ibid.: 132.

- subpallipes Tob. Ta: Janakkala. ibid.: 135.

- pulchriventris Fisch. $N$ : Nurmijärvi, St: Kokemäki. ibid.: 136.

- fuscipennis Wesm. Ab, N, Sa, Sb, Kb. ibid.: 137.

— padidalis Fisch. Sa: Ristiina. ibid.: 137.

— pulcherrimus Fisch. Sa: Mikkeli. ibid.: 137.

— staryi Fisch. Ta, Sa, Kb. ibid.: 138.

- crassipes Wesm. Al, Ab. ibid.: 139.
— mongolaltaiensis Fisch. N: Nurmijärvi. ibid.: 139.

— adentatus Fisch. N: Nurmijärvi. ibid.: 139.

- abditiformis Fisch. N, Sa, Kl. ibid.: 139.

- dureseaui Fisch. N: Nurmijärvi. ibid.: 140.

- funebris Wesm. Al, N, Ta, Sa, Sb, Om, Ok, Le. ibid.: 140.

- inflammatus Fisch. Ab, N, St, Sa, Sb, Kb, Ok. ibid.: 140.

- minusculae Fisch. N: Espoo. ibid.: 142.

- mirabilis Fisch. $N$ : Nurmijärvi, Sa: Mikkeli. ibid.: 142.

— opacus Fisch. N: Nurmijärvi. ibid.: 143.

- soenderupianus Fisch. Ab, N, Ka, Ta, Sa, Tb, $S b, K b, L k$. ibid.: 143.

- turcicus Fisch. Al, Kl, Ok. ibid.: 143.

Psyttalia concolor Szépl. Al: Lemland (A. Nordman), ibid.: 144.

- rhagoleticola (Sachtl.) N, Ta. ibid.: 144.

Eurytenes abnormis (Wesm.) Ta: Lammi. ibid.: 144.

- campanariae Fisch. $N$ : Nurmijärvi, Le: Kilpisjärvi. ibid.: 145.

- cubitalis Fisch. St: Reposaari (V. Lauro). ibid.: 145.

- impatientis Fisch. $N$ : Kirkkonummi. ibid.: 145.

- vockerothi Fisch. Al, N, Ok, Ks, Lk, Li. Fischer 1997: 182; Fischer \& Koponen 1999: 146.

Biosteres punctiscuta (Thoms.) Ab: Turku, $N$ : Helsinki. Fischer 1996 Zschr. Arb.Gem. Österr. Entomol. 48: 60; Fischer \& Koponen 1999: 148.

- colorativentris (Fisch.) Ab, N, Sa. Fischer \& Koponen 1999: 151.

- magnicornis (Wesm.) N, Ta. ibid.: 151.

Desmiostoma parvulum (Wesm.) N,Lk,Li. ibid.: 152.

Trachyusa aurora (Halid.) N, St, Ta. Koponen 1999 EF 10: 199.

- nigrothoracica Acht. \& O'C. N, St, Ta, Sa, $K l, K b$. ibid.: 199.

Adelurota florimela (Halid.) Al, Ab, N, Ka, Ta, $\mathrm{Sa}, \mathrm{Kl}, \mathrm{Tb}, \mathrm{Kb}, \mathrm{Ok}, \mathrm{Ob}, \mathrm{Ks}$. Koponen $2000 \mathrm{EF}$ 11: 239.

Anisocyrta alpinicola Acht. N: Helsinki. ibid.: 240.

- perdita (Halid.) Al, Ab, N, St, Ta, Sa, Tb, Kb, $O k, L k, L e, L i$. ibid.: 241.

Asyntactus rhogaleus Marsh. N, Ta, Lk. ibid.: 241.

Symphanes striolata (Thoms.) Ab, N, Sa, Tb. ibid.: 242.

Ibalia jakowlewi Jacobs. Sa: Juva. Martikainen \& Viitasaari 1996 Sahlbergia 3: 26. 
Torymus flavipes (Walk.) (dauci auct., viridissimus Boh.) sp.dist. de Vere Graham \& Gijswijt 1998 Zool. Verhand. 317: 97.

Pteromalus pontaniae Ask. Le: Kilpisjärvi. Kopelke 1994: 97

Coelopisthia caledonica Ask. Al: Finström. Lei et al. 1997: 637.

Eulonchetron scalprum (Ask.) Le: Kilpisjärvi. Kopelke 1994: 96.

Metaphycus nadius (Walk.) N: Hyvinkää (M. Koponen). Guerrieri \& Noyes 2000 System. Entomol. 25: 158.

- asterolecanii (Merc.) N: Nurmijärvi (M. Koponen), ibid.: 165.

- dispar (Merc.) N: Helsinki (M. Koponen), Ta: Lahti (M. Koponen). ibid.: 168.

- petitus (Walk.) Ab, N, Sb, Om. ibid.: 175.

- insidiosus (Merc.) Al, St, Tb. ibid.: 180.

- bulgaricus Sugonj. Ta, Om, Ok. ibid.: 186.

- roubali Hoff. Al: Jomala (W. Hellén), $N$ : Nurmijärvi (M. Koponen). ibid.: 195.

- unicolor Hoff. $N$ : Nurmijärvi (M. Koponen). ibid.: 195.

— punctipes (Dalm.) Ab: Nystad (W. Hellén). ibid.: 205.

*_annasor Guerr.\& N. Ta: Lammi (M. Koponen), ibid.: 206.
Syrphophagus sosius (Walk.) Sa: Ristiina (M. Koponen). Trjapitzin et al. 1995 Dipterol. Res. 6: 37.

Leptorhaptus docilis Nixon $K b$ : Ilomantsi. Humala 1995: 156.

- egregius Kieff. Kb: Ilomantsi. Humala 1995: 156.

Aclista prolongata Kieff. Kb: Ilomantsi. Humala 1995: 156

Pantoclis hirtistilus Kieff. Kb: Ilomantsi. Humala 1995: 156.

Zygota dentatipes (Kieff.) Kb: Ilomantsi. Humala 1995: 156.

Belyta pedestris Kieff. $K b$ : Ilomantsi. Humala 1995: 156.

— seron Nixon Finland. Maček 1996 Sborn. Nár. Muz. Praze, Ser. B 51: 15.

- borealis Whitt. (brevicornis auct.) Finland. ibid.: 20.

Chrysocharis pontaniae Ask. \& Kop. Le: Kilpisjärvi. Kopelke 1994: 93.

Spilomicrus crassiclavis Kieff. Kb: Tohmajärvi (M. Koponen). Notton 1999 Bull. Nat. Hist. Mus. (Ent.) 68: 133.

Bethylus boops (Thoms.) Ta: Janakkala. Vikberg 1999 EF 10: 246.

Trimorus therycides (Walk.) (autumnalis (Thoms.), 
pallipes (Thoms.)). Finland. Johnson 1992 Mem. Amer. Ent. Inst. 51: 552.

* Formica fennica Seif. Sb: Iisalmi, $K b$ : Kitee \& Ilomantsi. Seifert 2000 Zoosystema 22: 535. Spilomena differens Blüthg. (sp. dist.) $A l, A b, N$, St, Ta, Sa. Vikberg 2000 EF 11: 49.

*_valkeilai Vikb. Ab, N, Ta, Sa, Tb. ibid.: 43. Crossocerus assimilis (Smith) Ta: Janakkala. Vikberg 1999: 245.

Present number: $5755-84+305=5976$ spp.

In 1971, Hellén commented upon the increase in numbers of insect species during the past 50 years (Notulae Entomol. 51: 90), and made a prediction on how the situation might develop. It may be of interest to see how his suggestion for the year 2000 agrees with what we actually have. In Table 1, the number of species in various orders is given for the years 1950, 1970 and 2000, as well as Hellén's prediction for 2000 (column $2000 \mathrm{H})$. It should be kept in mind that Hellén's numbers also include those species, which only were collected in areas lost through the Second World War, and which accordingly are no longer considered part of the Finnish fauna.
Table 1. Number of insect of various orders reported from Finland in 1950, 1970 and 2000, together with Hellén's prediction $(2000 \mathrm{H})$ for the year 2000 .

\begin{tabular}{lrrrr}
\hline & 1950 & 1970 & $2000 \mathrm{H}$ & 2000 \\
\hline Collembola & 156 & 170 & 180 & 218 \\
Diplura & 1 & 1 & 2 & 1 \\
Protura & 1 & 3 & 5 & 3 \\
Thysanura & 4 & 4 & 4 & 4 \\
Ephemeroptera & 57 & 59 & 65 & 55 \\
Odonata & 50 & 51 & 53 & 52 \\
Plecoptera & 35 & 33 & 40 & 35 \\
Dermaptera & 2 & 2 & 3 & 2 \\
Orthoptera & 35 & 36 & 38 & 32 \\
Dictyoptera & 6 & 6 & 6 & 7 \\
Psocoptera & 51 & 56 & 65 & 65 \\
Phthiraptera & 147 & 223 & 368 & 292 \\
Hemiptera & 902 & 1130 & 1300 & 1376 \\
Thysanoptera & 113 & 113 & 120 & 114 \\
Neuroptera & 61 & 62 & 65 & 62 \\
Coleoptera & 3239 & 3412 & 3650 & 3632 \\
Strepsiptera & 6 & 7 & 10 & 5 \\
Trichoptera & 202 & 202 & 210 & 213 \\
Lepidoptera & 2071 & 2196 & 2350 & 2426 \\
Mecoptera & 6 & 6 & 8 & 6 \\
Diptera & 4098 & 4655 & 5400 & 5349 \\
Siphonaptera & 44 & 44 & 50 & 52 \\
Hymenoptera & 3700 & 4886 & 6200 & 5976 \\
Total & 14987 & 17357 & 20192 & 19977 \\
\hline
\end{tabular}

